\title{
The Kinetics of a Novel Cyclic Reaction System and its Use in the Assay of Polynucleotide Phosphorylase or Adenosine Diphosphate plus Adenosine Triphosphate
}

\author{
By R. J. H. WILSON AND M. D. LILLY \\ Department of Chemical Engineering (Biochemical Engineering Section), \\ University College London, Torrington Place, London W.C. 1
}

(Received 18 August 1967)

\begin{abstract}
1. A sensitive new method is described for the rapid optical assay of polynucleotide phosphorylase in crude bacterial extracts of low specific activity. 2. The assay is non-linear with time and a quick method for determining the approximate activity by using a nomograph is given. 3. The dependence of the assay on $\mathrm{Mg}^{2+}$ concentration was investigated and the conditions that gave first-order kinetics were determined. 4. The theoretical behaviour of the system was examined mathematically and shown to tend to a simple dynamic steady state, under certain conditions. 5. The potential uses of such cyclic systems are discussed.
\end{abstract}

The measurement of polynucleotide phosphorylase (EC 2.7.7.8) in crude extracts from bacterial suspensions has been complicated by the occurrence of various side reactions involving substrates or products (Grunberg-Manago, 1963). Polynucleotide phosphorylase catalyses the polymerization of ribonucleoside $5^{\prime}$-diphosphates to form polyribonucleic acids and free inorganic phosphate. The reaction is freely reversible and previous assay methods have used the formation or disappearance of one of the three reactants to measure the enzymic activity. Another type of assay depends on the ability of the enzyme to catalyse an exchange reaction between ribonucleoside 5 '-diphosphates and inorganic phosphate that may be followed by using ${ }^{32} \mathrm{P}$.

The relationship between the exchange assay and other assays involving the polymerization or depolymerization reaction is not clear and certainly varies with the bacterial origin of the enzyme (Williams \& Grunberg-Manago, 1964). The exchange reaction is the only one with which other known enzymic reactions likely to occur in crude extracts do not interfere, and has therefore been widely used during purification procedures. When used in this way it has serious disadvantages in that it is tedious to perform and takes a long time to complete. The present paper reports the development of an optical assay method that overcomes the problems of side reactions in most cases but that nevertheless is reasonably quick. This assay is based on a cyclic system giving a steadily accelerating rate of reaction during the assay, enabling much lower enzyme activities to be measured.

\section{THEORETICAL}

The assay is based in part on the optical assay method of Ochoa \& Mii (1961), in which the production of ADP from polyadenylic acid is measured directly by the use of a coupled pyruvate kinase (EC 2.7.1.40)-lactate dehydrogenase (EC 1.1.1.27) system. Polynucleotide phosphorylase activity is measured as the rate of decrease of extinction at $340 \mathrm{~m} \mu$ :

$$
\begin{aligned}
& \text { Polyadenylic acid }+n \mathrm{P}_{1} \stackrel{\mathrm{Mg}^{2+}}{\longrightarrow} n \mathrm{ADP} \\
& \text { Phosphoenolpyruvate }+\mathrm{ADP} \rightarrow \text { pyruvate }+\mathrm{ATP} \\
& \text { Pyruvate }+\mathrm{NADH}+\mathrm{H}^{+} \rightarrow \text { lactate }+\mathrm{NAD}^{+}
\end{aligned}
$$

This assay is linear for most of its course under the conditions set out by Ochoa \& Mii (1961), indicating essentially zero-order kinetics for the phosphorylase reaction and first-order kinetics for the two indicator reactions. Its inapplicability to crude extracts has been largely due to direct oxidation of NADH by an oxidase system present in cell extracts. In the above system production of one molecule of ADP leads to the oxidation of only one molecule of NADH. In the present assay we have attempted to improve the sensitivity of the system by recycling the ATP formed; this is achieved by adding creatine kinase (EC 2.7.3.2) and creatine to the assay:

$$
\text { Creatine+ATP } \stackrel{\mathrm{Mg}^{2+}}{\longrightarrow} \text { creatine phosphate + ADP }
$$

This modification causes the rate of oxidation of NADH to accelerate during the assay. The trace from the recording spectrophotometer is therefore non-linear (Fig. 1).

Assuming a state of dynamic equilibrium and first-order kinetics, the rate of cycling can be shown to be:

$$
v=\frac{k_{2} k_{3}}{k_{2}+k_{3}} \cdot[\mathrm{A}]=K \cdot[\mathrm{A}]
$$




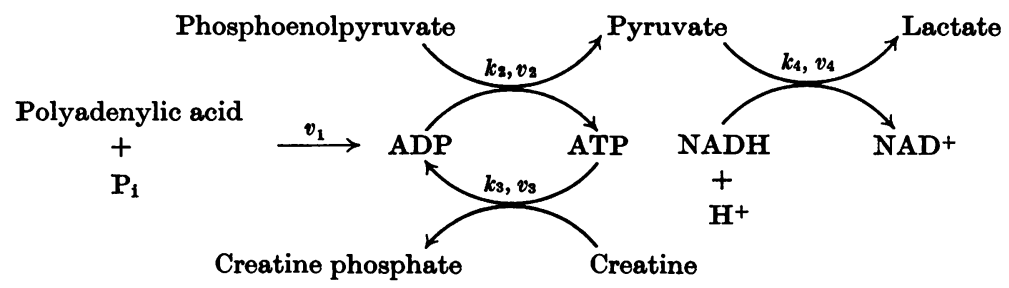

Scheme 1.

where $k_{2}$ and $k_{3}$ are the first-order rate constants for the reactions catalysed by pyruvate kinase and creatine kinase respectively, and [A] is the total concentration of $\mathrm{ADP}+$ ATP present in the system. The velocity of the cycle in dynamic equilibrium is therefore proportional to [A], which will increase with time, $t$, as the polynucleotide phosphorylase reaction proceeds:

$$
[\mathrm{A}]_{t}=v_{1} t
$$

where $v_{1}$ is the maximal velocity of the phosphorylase reaction. Hence:

$$
v=K v_{1} t
$$

Integrating, the total substrate throughput of each arm of the cycle after time $t$ is:

$$
\frac{1}{2} K v_{1} t^{2}
$$

Thus the total amount of NADH oxidized in the indicator reaction should be proportional to $t^{2}$. A knowledge of $K$ would then allow $v_{1}$ to be calculated. This simple treatment is not strictly correct, however, since it assumes a state of dynamic equilibrium at all times, and this is clearly not so as it would require an infinitely rapid recycling of the ATP.

Evaluating without this assumption then, for the reactions shown in Scheme 1 , where $v_{1}$ is constant, $v_{2}=k_{2}$ [ADP], $v_{3}=k_{3}[\mathrm{ATP}], v_{4}=k_{4}$ [pyruvate] and [A] $=$ $[\mathrm{ADP}]+[\mathrm{ATP}]$, it can be seen that:

$$
\frac{\mathrm{d}[\mathrm{ADP}]}{\mathrm{d} t}=v_{1}+v_{3}-v_{2}, \frac{\mathrm{d} v_{2}}{\mathrm{~d} t}=k_{2} \cdot \frac{\mathrm{d}[\mathrm{ADP}]}{\mathrm{d} t}
$$

and $\quad[\mathrm{A}]=[\mathrm{A}]_{0}+v_{1} t$

where $[A]_{0}=[A]$ at $t=0$, from which:

$$
\frac{\mathrm{d} v_{2}}{\mathrm{~d} t}=k_{2} k_{3} v_{1} t-\left(k_{2}+k_{3}\right) v_{2}+k_{2}\left(k_{3}[\mathrm{~A}]_{0}+v_{1}\right)
$$

Eqn. (3) is a particular case of the standard linear first-order differential equation:

$$
\frac{\mathrm{d} v_{2}}{\mathrm{~d} t}+P(t) v_{2}=Q(t)+\text { constant }
$$

which may be integrated by multiplying both sides by the integrating factor e $\int \mathrm{P}(t) \mathrm{d} t$ (in this case e $\int_{0}^{t}\left(k_{2}+k_{3}\right) \mathrm{d} t$, from which we can obtain:

$$
v_{2} \mathrm{e}^{\left(k_{2}+k_{3}\right) t}=\int_{0}^{t}\left[\left(k_{2} k_{3} v_{1} t+k_{2}\left(k_{3}[\mathrm{~A}]_{0}+v_{1}\right)\right] \mathrm{e}^{\left(k_{2}+k_{2}\right) t} \cdot \mathrm{d} t\right.
$$

Integration of this equation by parts gives:

$$
v_{2}=K v_{1} t+K[\mathrm{~A}]_{0}+K\left(\frac{1}{k_{3}}-\frac{1}{k_{2}+k_{3}}\right) v_{1}+C_{1} \mathrm{e}^{-\left(k_{3}+k_{3}\right) t}
$$

Where $C_{1}$ is a constant, whose value may be determined since when $t=0, v_{2}=K[\mathrm{~A}]_{0}$ (see eqn. 1 ):

$$
C_{1}=-\frac{K^{2}}{k_{3}^{2}} v_{1}
$$

Inserting this value, and rearranging:

$$
v_{2}=K v_{1} t+\frac{K^{2} v_{1}}{k_{3}^{2}}\left(1-\mathrm{e}^{-\left(k_{2}+k_{3}\right) t}\right)+K[\mathrm{~A}]_{0}
$$

Considering the indicator reaction; since:

$$
\frac{\mathrm{d}[\text { pyruvate }]}{\mathrm{d} t}=v_{2}-v_{4} \text { and } \frac{\mathrm{d} v_{4}}{\mathrm{~d} t}=k_{4} \cdot \frac{\mathrm{d}[\text { pyruvate }]}{\mathrm{d} t}
$$

then :

$$
v_{4}=\int_{0}^{t} k_{4}\left(v_{2}-v_{4}\right) \cdot \mathrm{d} t
$$

Inserting eqn. (4) into eqn. (5) and integrating in the same way as for eqn. (3), then:

$$
\begin{gathered}
v_{4}=K v_{1} t+K[\mathrm{~A}]_{0}+\frac{K^{2} v_{1}}{k_{3}{ }^{2}}\left\{1-\frac{k_{4}}{k_{4}-k_{2}-k_{3}} \cdot \mathrm{e}^{-\left(k_{2}+k_{3}\right) t}\right\} \\
-\frac{K v_{1}}{k_{4}}\left\{1-\frac{\left(k_{2}+k_{3}\right)\left(k_{4}-k_{3}\right)}{\left(k_{4}-k_{2}-k_{3}\right) k_{3}} \cdot \mathrm{e}^{-k_{4} t}\right\}
\end{gathered}
$$

The observed change in NADH concentration, $\Delta$ [NADH], is given by:

$$
\begin{aligned}
\Delta[\mathrm{NADH}] & =-\int_{0}^{t} v_{4} \cdot \mathrm{d} t=[\mathrm{NADH}]_{0}-[\mathrm{NADH}]_{t} \\
= & \frac{1}{2} K v_{1} t^{2}+K[\mathrm{~A}]_{0} t+K v_{1}\left\{\frac{k_{2}}{k_{3}\left(k_{2}+k_{3}\right)}-\frac{1}{k_{4}}\right\} t \\
& +\frac{k_{2}{ }^{2} k_{4} v_{1}\left[\mathrm{e}^{-\left(k_{2}+k_{3}\right) t}-1\right]}{\left(k_{2}+k_{3}\right)^{3}\left(k_{4}-k_{2}-k_{3}\right)} \\
& +\frac{k_{2}\left(k_{4}-k_{3}\right)\left(1-\mathrm{e}^{-k_{4} t}\right) v_{1}}{k_{4}{ }^{2}\left(k_{4}-k_{2}-k_{3}\right)}
\end{aligned}
$$

Eqn. (7) may be rearranged and condensed to give:

$$
\begin{gathered}
\frac{\Delta[\mathrm{NADH}]}{t}=\frac{1}{2} K v_{1} t+K[\mathrm{~A}]_{0}+B v_{1}+\frac{v_{1} C}{t}\left(\mathrm{e}^{-\left(k_{2}+k_{3}\right) t}-1\right) \\
+\frac{v_{1} D}{t}\left(1-\mathrm{e}^{-k_{4} t}\right)
\end{gathered}
$$

Thus a plot of $\Delta[\mathrm{NADH}] / t$ against $t$ will tend to a straight line of slope $\frac{1}{2} K v_{1}$, as the last two terms approach zero, and $v_{1}$ may be calculated if $K$ is known. The approach to linearity of eqn. (8) may be examined by differentiating the equation to give the slope of the curve: 


$$
\begin{aligned}
\frac{1}{v_{1}} \cdot \frac{\mathrm{d}(\Delta[\mathrm{NADH}] / t)}{\mathrm{d} t} & =\frac{1}{2} K+\frac{C}{t^{2}}\left\{1-\left[1+\left(k_{2}+k_{3}\right) t\right] \cdot \mathrm{e}^{-\left(k_{2}+k_{3}\right) t}\right\} \\
& -\frac{D}{t^{2}}\left[1-\left(1+k_{4} t\right) \mathrm{e}^{-k_{4} t}\right]
\end{aligned}
$$

The right-hand side of this equation has been computed for various values of $k_{2}, k_{3}, k_{4}$ and $t$, and the results have been plotted against $t$ on a $\log$-log basis (Fig. 2), to show the approach to the steady state of the multi-component system. When the curves in Fig. 2 reach a steady value equal to $\frac{1}{2} K$ the relationship between $\Delta[\mathrm{NADH}] / t$ and $t$ becomes linear. The time taken to reach this condition is dependent on the parameters $k_{2}, k_{3}, k_{4}$ and $K$, but not on $v_{1}$. The value of $K$ affects the system in two ways. First, the value of $K$ partly determines the slope under steadystate conditions so that a high value of $K$ increases the sensitivity of the assay. Secondly, the approach to linearity is in most cases quicker at high values of $K$. For a given value of $K$ the time taken to reach linearity varies considerably according to the relative values of $k_{2}$ and $k_{3}$, and particularly with $k_{4}$, which should be greater than both $k_{2}$ and $k_{3}$, preferably by a single order of magnitude. An increase in $k_{4}$ above this level has little effect. For a given value of $k_{4}(>1)$ the rate of approach to the steady state is increased slightly with increasing $k_{2}$, more rapidly with increasing $k_{3}$ and fastest of all when $k_{3}$ is much greater than $k_{2}$.

From the point of view of a practical assay system, economical use of enzymes is important. With $\mathrm{K}$ about $1 \mathrm{~min} .^{-1}$ and $k_{4}$ about $10 \mathrm{~min} .^{-1}$ the plot of $\Delta[\mathrm{NADH}] / t$ against $t$ will become linear after 1-2 min. This was considered to be sufficiently rapid for the present system and the above values were used in practice.

\section{MATERIALS AND METHODS}

Reagents. Tris, creatine, NADH, polyadenylic acid (potassium salt), lactate dehydrogenase [ox heart, as suspension in $\left(\mathrm{NH}_{4}\right)_{2} \mathrm{SO}_{4}, 15 \mathrm{mg}$. $/ \mathrm{ml}$., activity 150 units $/ \mathrm{mg}$.] and pyruvate kinase [rabbit skeletal muscle, as suspension in $\left(\mathrm{NH}_{4}\right)_{2} \mathrm{SO}_{4}, 2 \mathrm{mg} . / \mathrm{ml}$., activity $110 \mathrm{units} / \mathrm{mg}$.] were obtained from Koch-Light Laboratories Ltd., Colnbrook, Bucks. Phosphoenolpyruvate (tricyclohexylammonium salt) and creatine kinase (crystallized from rabbit muscle, activity 20 units/mg.) were obtained from Calbiochem Ltd., London, W. 1. ATP (horse muscle, $99 \%$ pure) was supplied by Sigma Chemical Co., St Louis, Mo., U.S.A. All other chemicals were A.R. grade where available. Glass-distilled water was used throughout.

Growth of Azotobacter vinelandii. A. vinelandii N.C.I.B. 8789, maintained by subculture on to nitrogen-free sucroseagar every 2 months, was grown in Burk's nitrogen-free medium, $\mathrm{pH} 7 \cdot 3$, which contained (g./l.): sucrose, 20; $\mathrm{K}_{2} \mathrm{HPO}_{4}, 0.64 ; \mathrm{KH}_{2} \mathrm{PO}_{4}, 0 \cdot 16 ; \mathrm{NaCl}, 0 \cdot 2 ; \mathrm{MgSO}_{4}, 7 \mathrm{H}_{2} \mathrm{O}$, $0.2 ; \mathrm{CaSO}_{4}, 2 \mathrm{H}_{2} \mathrm{O}, 0.05 ; \mathrm{Na}_{2} \mathrm{MoO}_{4}, 0.0025$; ferric citrate, 0.018 . A culture $(100 \mathrm{ml}$.) grown with continuous aeration and agitation in a $20 \mathrm{oz}$. bottle for $30 \mathrm{hr}$. at $30^{\circ}$ was used to inoculate 21. of the same medium and incubated in the same way for $24 \mathrm{hr}$. This culture was then transferred to $200 \mathrm{l}$. of the same medium, vigorously aerated and maintained at $28^{\circ}$ and $\mathrm{pH} \mathrm{7.3}$. At the end of the growth phase (about $30 \mathrm{hr}$.) the bacteria were harvested in a $6 \mathrm{P}$ Pressurtite supercentrifuge (Sharples Co., Camberley, Surrey). The yield of bacteria was about $1 \cdot 1 \mathrm{~kg}$. wet wt.
Preparation of polynucleotide phosphorylase. A crude preparation of polynucleotide phosphorylase was made from $A$. vinelandii by a slight modification of the method of Ochoa \& Mii (1961). The product from stage 2 was stored at $-15^{\circ}$. This preparation had an activity of $0.013 \mu \mathrm{mole}$ of ADP formed $/ \mathrm{min} . / \mathrm{mg}$. of protein at $30^{\circ}$, as measured in the assay described below. It also showed an NADH oxidase activity of about $0.009 \mu$ mole of $\mathrm{NADH}$ oxidized/min./mg. of protein in the same assay.

Preparation and storage of assay system. The components of the assay were made up as five separate solutions as follows. For reagent $A$ a solution was made by mixing $0.6 \mathrm{M}$-tris (50 ml.), $30 \mathrm{~mm}$-EDTA (10 ml.), $0.3 \mathrm{M}-\mathrm{KH}_{2} \mathrm{PO}_{4}$ $(10 \mathrm{ml}$.$) and water \left(18 \mathrm{ml}\right.$.) and adjusting to $\mathrm{pH} 8.1$ at $30^{\circ}$ with $5 \mathrm{~N}-\mathrm{HCl}$. Solid phosphoenolpyruvate $(223.5 \mathrm{mg}$.), lactate dehydrogenase $(1 \mathrm{ml}$.$) , pyruvate kinase (1 \mathrm{ml}$.$) and$ creatine kinase $(10.3 \mathrm{mg}$.) were added and the whole mixture was stored in $10 \mathrm{ml}$. portions at $-15^{\circ}$. Reagent $B$ was made by mixing $75 \mathrm{~mm}-\mathrm{MgSO}_{4}(20 \mathrm{ml}$.) and $48 \mathrm{~mm}$-creatine $(150 \mathrm{ml}$.$) and stored at 2^{\circ}$. At this temperature some creatine crystallizes out, but it redissolves when the solution is warmed before assay. Reagent $C$ was a solution of polyadenylic acid (1 mg./ml.) made up in water and stored in $1 \mathrm{ml}$. portions at $-15^{\circ}$. Reagent $D$ was $0.15 \mathrm{~mm}$-ATP in water, stored in $2 \mathrm{ml}$. portions at $-15^{\circ}$. Reagent $E$ was $3 \mathrm{mM}-\mathrm{NADH}$ made up in water previously adjusted to about $\mathrm{pH} 10$ with $\mathrm{KOH}$. This solution was normally made on the day of the assay.

Assay procedure. The following reagent solutions (at $30^{\circ}$ ) were pipetted into a $3 \mathrm{ml}$. silica cuvette $(1 \mathrm{~cm}$. light-path) maintained at $30^{\circ}: A, 1.7 \mathrm{ml}$; $B, 0.9 \mathrm{ml}$; $C, 0.1 \mathrm{ml}$.; $E$, $0.2 \mathrm{ml}$; p polynucleotide phosphorylase preparation, $0.1 \mathrm{ml}$. After allowing time for equilibration of the other reagents the reaction was started by the addition of either the enzyme or the polyadenylic acid. The reaction was followed at $340 \mathrm{~m} \mu$ in a Hilger-Gilford reaction-kinetics spectrophoto-

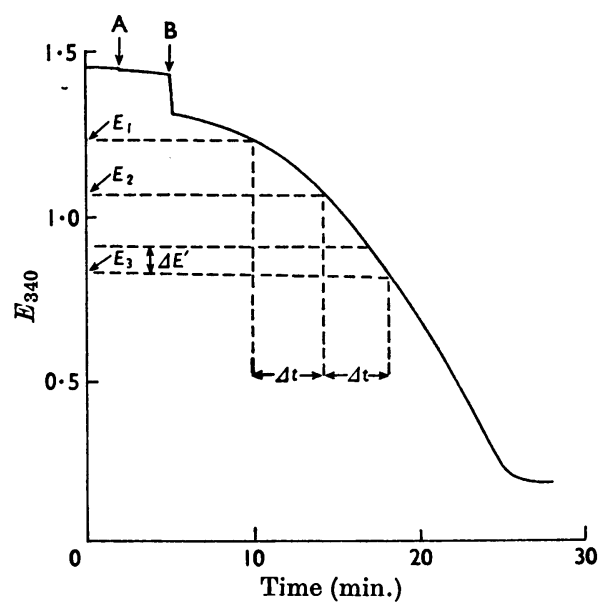

Fig. 1. Typical recorder trace in the assay of polynucleotide phosphorylase. The enzyme extract was added at point $A$, and the polyadenylic acid solution at point $B$ (see section on the calculation of $v_{1}$ in the Materials and Methods section). 

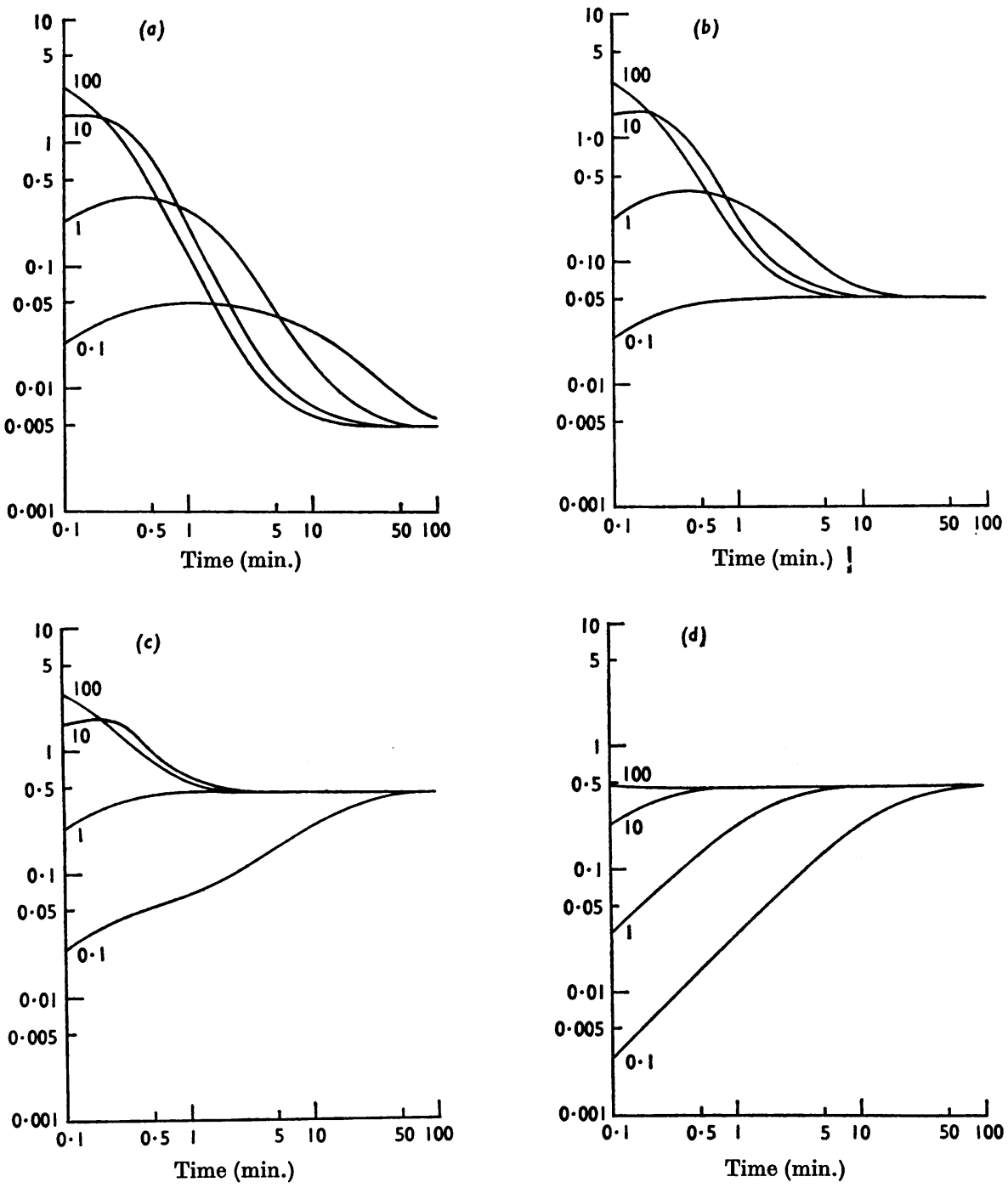

Fig. 2. Theoretical curves for the approach of $\Delta[\mathrm{NADH}] / t$ versus $t$ to a linear relationship. The following values were used:

$\begin{array}{cccc} & K\left(\text { min. }^{-1}\right) & k_{2}\left(\min ^{-1}\right) & k_{3}\left(\min ^{-1}\right) \\ (a) & 0.01 & 10.00 & 0.01 \\ (b) & 0.10 & 10.00 & 0.10 \\ (c) & 0.91 & 10.00 & 1.00 \\ (d) & 0.91 & 1.00 & 10.00\end{array}$

In each case curves for $k_{4}=0 \cdot 1,1,10$ and $100 \mathrm{~min}^{-1}$ are shown.

The ordinate is $\frac{1}{v_{1}} \cdot \frac{\mathrm{d}(\Delta[\mathrm{NADH}] / t)}{\mathrm{d} t}$, calculated from the right-hand side of eqn. (9).

meter. A control assay with ATP (reagent $D, 0.2 \mathrm{ml}$.) replacing the enzyme and polyadenylic acid was normally carried out before each set of assays, to determine the value of $K$. The trace in this case is linear and the slope proportional to $K$.
Effect of $\mathrm{Mg}^{2+}$ concentration on the assay. The assay procedure as outlined for the determination of $K$ was used except that reagent solution $B$ was replaced by $48 \mathrm{mM}$ creatine $\left(0.7 \mathrm{ml}\right.$.) and various concentrations of $\mathrm{MgSO}_{4}$ in $0.2 \mathrm{ml}$. 


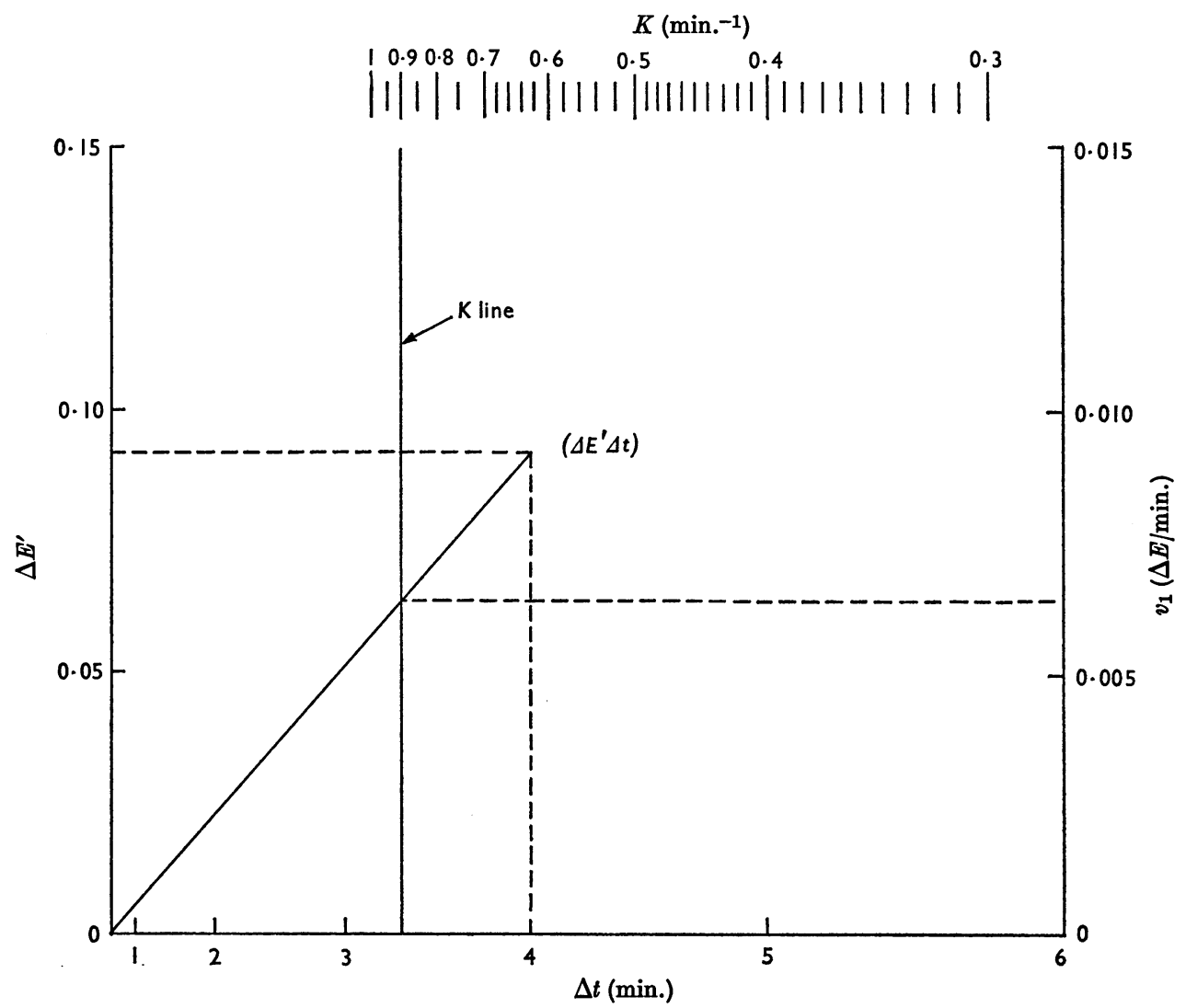

Fig. 3. Nomograph for the rapid calculation of an approximate value of $v_{1}$, obtained for $K 0.9 \mathrm{~min} .^{-1}$ from the values of $\Delta t$ and $\Delta E^{\prime}$ shown in Fig. 1. Details are given in the section on calculation of $v_{1}$ in the Materials and Methods section.

Calculation of polynucleotide phosphorylase activity $\left(v_{1}\right)$. For accurate determinations of $v_{1}, \Delta E_{340} / t$ was plotted against $t$. After 3-4 min. the values fall on a straight line with a slope equal to $\frac{1}{2} K v_{1}$. The value of $v_{1}$, expressed in terms of $\mu$ moles of ADP formed/min./ml., may be calculated by dividing by $\frac{1}{2} K$ (previously determined) and then using a conversion factor relating the extinction values to $\mu$ moles of NADH $\left(0 \cdot 1613 \mathrm{~mm}-\mathrm{NADH}\right.$ has $\left.E_{340}^{1 \mathrm{~cm}} \cdot 1 \cdot 0\right)$.

A simpler rapid method has been devised for calculating approximate values of $v_{1}$ from the original curve obtained for extinction at $340 \mathrm{~m} \mu$ (see Fig. 1). The increase in the change of extinction $\left[\Delta E^{\prime}=\left(E_{2}-E_{3}\right)-\left(E_{1}-E_{2}\right)\right]$ is measured by taking the difference between the changes of extinction over two adjacent and equal time-intervals $(\Delta t)$ on the recorder trace. (A pair of dividers may conveniently be employed for this purpose.) The time-interval chosen (2-6min.) depends on the magnitude of $v_{1}$. Time must be allowed for the system to come to dynamic equilibrium, as indicated in the Theoretical section (generally about 3-4min.). Since it can be shown that $\Delta E^{\prime}=K v_{1} \Delta t^{2}$, it is possible to construct a nomograph (Fig. 3) so that, by using the previously determined value of $K$, plotting $\Delta E^{\prime}$ for the $\Delta t$ chosen and drawing a line through this point and the origin, $v_{1}$ can be read off in terms of $\Delta E / \min$. This value may then be converted into $\mu$ moles/min./ml. by multiplying by the conversion factor $(0 \cdot 1613)$. The procedure may be summarized thus. (1) A vertical line (the $K$ line) is drawn through the predetermined value of $K$. (2) $\Delta E^{\prime}$ is plotted for the appropriate value of $\Delta t$ and a line is drawn from this point through the origin; (3) $v_{1}$ is read off at the point where this line cuts the $K$ line. A more accurate answer can be determined later by the graphical method.

\section{RESULTS}

Effect of ATP concentration on the kinetics of the cyclic system. The variation of the reaction velocity of the cyclic system with added ATP concentration is shown in Fig. 4. The results, plotted on a log-log basis, show that first-order kinetics within the cycle are obeyed up to about $0.02 \mathrm{~mm}$-(ATP + ADP). At this concentration the rate of change of extinction is about $0 \cdot 1$ unit $/ \mathrm{min}$. In the assay of polynucleotide phosphorylase this rate is unlikely to be reached unless excessive amounts of the enzyme are used.

Determination of $K$. The value of $K$ for each new 


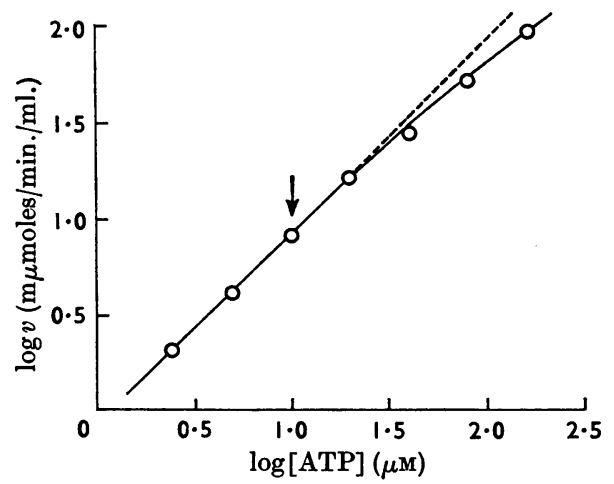

Fig. 4. Variation of the rate $(v)$ of the cyclic enzyme system with ATP concentration. The theoretical curve for firstorder kinetics assuming a value of $0.817 \mathrm{~min} .^{-1}$ for $K$ is shown by the broken line. The arrow indicates the ATP concentration normally used to determine $K$.

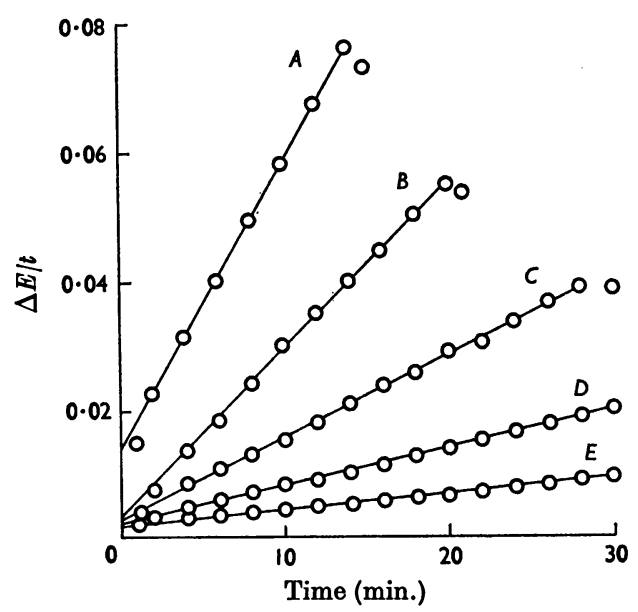

Fig. 5. Relationship between $\Delta E / t$ and $t$ for assays in which the following volumes ( $\mu \mathrm{l}$.) of polynucleotide phosphorylase solution were used: $A, 50 ; B, 25 ; C, 12.5 ; D, 6.25 ; E, 3 \cdot 125$. Experimental details are given in the text.

reagent solution was determined by using a concentration of $0.01 \mathrm{~mm}$-ATP, and normally found to be about $0 \cdot 8-0.9 \mathrm{~min} .^{-1}$ when calculated from the equation $v=K[\mathrm{ATP}]$. Freezing and storage of reagent $A$ for several months resulted in little or no loss in activity.

Determination of $v_{1}$. A typical trace from the recorder is shown in Fig. 1. If there is any ADP or ATP present in the polyadenylic acid or enzyme solutions or if there is NADH oxidase activity present in the enzyme preparation, then there will be a steady rate superimposed on the trace. In the

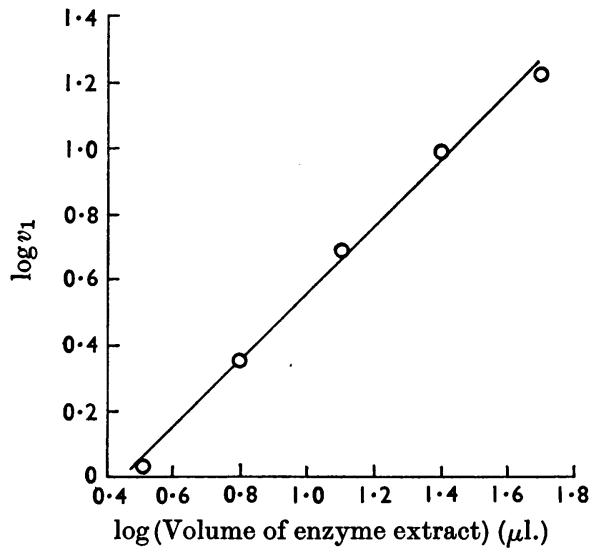

Fig. 6. Relationship between $v_{1}$ and concentration of polynucleotide phosphorylase for values obtained from Fig. 5 . The units of $v_{1}$ are $10^{-10} \mathrm{~mole} / \mathrm{min} . / \mathrm{ml}$. of assay solution. The slope of the line $\left(45^{\circ}\right)$ indicates that $v_{1}$ is linearly proportional to the enzyme concentration over the range measured; see the text for further details.

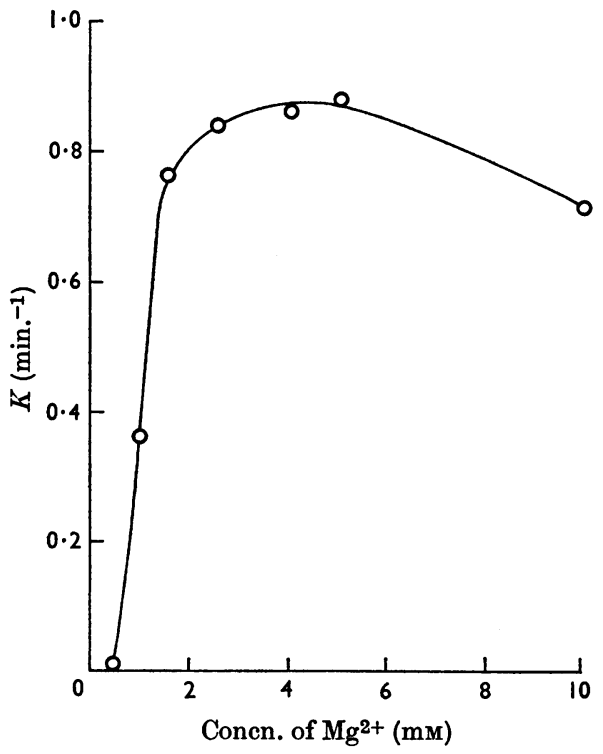

Fig. 7. Variation of $K$ with $\mathrm{Mg}^{2+}$ concentration. Experimental details are given in the text.

subsequent plot of $\Delta E / t$ against $t$ (Fig. 5) this results in the line intercepting the vertical axis at a higher point. The values of $v_{1}$ for the various enzyme concentrations used in Fig. 5 are given in Fig. 6 and show that the assay is linear with enzyme activity over the range $0 \cdot 1-1 \cdot 7 \mathrm{~m} \mu \mathrm{moles} / \mathrm{min} . / \mathrm{ml}$. 
in the cuvette. This corresponds to activities in the original solutions of $3-50 \mathrm{~m} \mu \mathrm{moles} / \mathrm{min} . / \mathrm{ml}$. under the conditions of the standard assay.

Effect of $\mathrm{Mg}^{2+}$ concentration on the assay. The value of $K$ was determined at various concentrations of $\mathrm{Mg}^{2+}$ in the assay. The results (Fig. 7) show that there is an absolute requirement for the cation with an optimum concentration of about $5 \mathrm{~mm}$. The EDTA in the reaction mixture appears to decrease considerably the effective concentration of $\mathrm{Mg}^{2+}$, especially at concentrations below $1 \mathrm{~mm}$. The $\mathbf{M g}^{2+}$ requirement of the polynucleotide phosphorylase preparation from $A$. vinelandii was not investigated.

\section{DISCUSSION}

The use of cyclic systems for enzyme and substrate assays has been limited in the past to systems resulting in linear rate curves, which are simply analysed, so that there is no problem involved in calculating the value of the unknown enzyme activity or substrate concentration. Such systems have been used therefore to assay either the cycling component (as the sum of its two forms) or one of the reactions making up the cycle. In the latter case the purpose of the cycle may be merely to remove an inhibitory product, but it will serve also to maintain the concentration of one substrate constant during the reaction. It is in the measurement of small quantities of substrate that this type of assay system is extremely useful. The total change measured may be increased by 100 -fold or more, depending on the time allowed for the assay. This fact has been the basis of techniques for the microanalysis of a very few substrates, which are suitable for cycling systems only by virtue of involving enzymes with the necessary kinetic properties. Where the requirements are satisfied the arrangement has proved a powerful analytical tool.

Probably the first successful use of such a system was the determination of $\mathrm{NAD}^{+}+\mathrm{NADH}$ by Jandorf, Klemperer \& Hastings (1941). They used a crude muscle extract as a source of the enzymes comprising the glycolytic pathway, and primed the system with glucose 1,6-diphosphate. Evolution of carbon dioxide was used as a measure of the total nicotinamide nucleotides. Quantities of about $1 \mu \mathrm{g}$. were measurable even in this crude assay system. The increased commercial availability of pure enzymes now allows cyclic systems of less complexity and greater flexibility, reliability and definition to be devised. Most cyclic systems so far used have been coupled to suitable indicator reactions resulting in spectral changes that may be followed in a recording spectrophotometer. Glock \& McLean (1955) followed the reduction of cytochrome $c$ at $550 \mathrm{~m} \mu$ in two systems that they devised for assaying separately the two nucleotide pairs $\mathrm{NAD}^{+}+\mathrm{NADH}$ and $\mathrm{NADP}^{+}+\mathrm{NADPH}$. In their systems the indicating reactions were conveniently doubling as single arms of the cycles involved, but this has not been achieved in any other system of this type. These two pairs of substrates have themselves been used in indicator reactions for the assay of ADP + ATP as a relatively large fall in $E_{340}$ occurs during the oxidation of $\mathrm{NADH}$. The enzymes involved have included glucose 6-phosphate dehydrogenase (Oliver, 1954; Cori, Traverso-Cori, Lagarrigue \& Marcus, 1958) and lactate dehydrogenase (Tanzer \& Gilvarg, 1959). For the arms of the cycle in ADP + ATP assays three enzymes have been employed in three possible combinations: hexokinase + pyruvate kinase (Grisolia, Mokrasch \& Hospelhorn, 1958); hexokinase+ creatine kinase (Oliver, 1954, to measure creatine kinase ; Chappell \& Perry, 1954) ; creatine kinase and pyruvate kinase (Tanzer \& Gilvarg, 1959). The phosphorylation of ADP with creatine kinase is a reversible reaction and it is essential therefore to operate the system under conditions that favour the desired reaction, i.e. a suitable $\mathrm{pH}$ and excess of the non-cycling substrates for both enzymes in the cycle.

In addition to these systems Perry (1954) coupled creatine kinase with myofibrillar adenosine triphosphatase during investigations of possible cell mechanisms involved in phosphate transfer. Cyclic systems may also be devised that dispense with the need for at least one of the enzymes of the cycle, the necessary reaction being spontaneous. Greenbaum, Clark \& McLean (1965) measured $\mathrm{NAD}^{+}+\mathrm{NADH}$ by using alcohol dehydrogenase or glucose 6-phosphate dehydrogenase to reduce $\mathrm{NAD}^{+}$, reoxidizing it non-enzymically with an electron carrier, phenazine methosulphate. This was then reoxidized from its reduced state by molecular oxygen, again in a spontaneous reaction. Oxygen uptake rate as measured with an oxygen electrode was found to give a measure of total coenzyme present.

A noteworthy case of a cyclic system catalysed on both arms by a single enzyme was discovered accidentally by Tietz \& Ochoa (1958) during attempts to isolate fluorokinase from rabbit skeletal muscle. They tried to assay the enzyme by measuring the formation of ADP from ATP and fluoride with the coupled system of pyruvate kinase and lactate dehydrogenase. Their results indicated that the pyruvate kinase also possessed fluorokinase activity, although this was to some extent masked by inhibition of the fluorokinase activity by phosphoenolpyruvate. Prompted by this observation they further discovered that their fluorokinase possessed pyruvate kinase activity, the ratio of activities proving the same at all stages of purification, indicating that both reactions are catalysed 
by the same enzyme. ATP (or ADP) can thus be considered to be a true cofactor in this single-enzyme cycle, the net reaction of which is:

Phosphoenolpyruvate $+\mathrm{F}^{-} \stackrel{\text { ATP }}{\longrightarrow}$

phosphorofluoridate + pyruvate

The kinetic constants of the enzymes chosen for a cyclic enzyme assay system must lie within fairly well-defined limits if the system is to give results from which the unknown component can be calculated easily. Where a component of one arm of the cycle is being assayed the limit is reached when the other arm of the cycle can no longer supply substrate at a rate sufficient to saturate the enzyme in question. However, in the present assay the concentrations of ADP and ATP must always be sufficiently below the $K_{m}$ values of the respective enzymes in the cycle that first-order kinetics are obeyed and the results are solvable as described in the Theoretical section. If the $K_{m}$ of one of these enzymes is high the overall reaction rate will be very low and the assay system insensitive. For maximum sensitivity with economical amounts of the enzymes involved, the enzymes in the cycle should have $K_{m}$ values for the cycling substrates that are low and of the same order. Pyruvate kinase and creatine kinase fulfil these requirements under certain conditions, both exhibiting $K_{m}$ values of about $10^{-4} \mathrm{M}$ in the directions involved (McQuate \& Utter, 1959; Kuby, Noda \& Lardy, 1954). The requirements for indicator reactions are not so exacting; it is only necessary to ensure that there is sufficient enzyme present to keep the intermediate substrate concentration low, and hence maintain the first-order nature of the reaction. A dynamic equilibrium will then be set up, as exemplified by the curves in Fig. 2. The indicator reaction should be virtually unidirectional under the conditions of the assay. Lactate dehydrogenase fulfils these requirements, having $K_{m}$ about $10^{-4} \mathrm{M}$ for pyruvate and being, in practice, irreversible unless forced by removal of product since it has an equilibrium constant very much in favour of lactate formation (Hakala, Glaid \& Schwert, 1956).

The increase in sensitivity gained by a system of the type reported here could conceivably be applied to any other enzyme producing ADP or ATP from any substrate other than these two, and where assay is difficult owing to low activity or interfering reactions of certain kinds. Chappell \& Perry (1954) found that, if they replaced hexokinase and glucose with myokinase and AMP in a cyclic system employing creatine kinase as the second arm, then in the presence of catalytic quantities of ADP or ATP the AMP could be converted into ADP, and hence creatine phosphate into creatine (which they measured). This reaction proceeds progressively faster since for each mole of ATP removed from the system 2 moles of ADP are added. Thus myokinase could have been assayed by the acceleration technique described above. In fact Chappell \& Perry (1954) only tested the system with either high ATP concentrations or with no ATP or ADP added at all, plus AMP as substrate. Consequently in the first instance a straight line was obtained for creatine formation, and in the second a curve resembling those described in this paper for polynucleotide phosphorylase assay, indicating the presence of initial catalytic quantities of ADP or ATP or both. Small quantities of AMP may be measured by reintroducing the hexokinase and glucose: AMP will be converted totally in time into ADP and a plot tending to linearity will result. Again catalytic amounts of ADP + ATP must be present to prime the cycle. These authors also found a requirement for $\mathbf{M g}^{2+}$ in their system, which closely mirrors that shown in Fig. 7 for the creatine kinase-pyruvate kinase cycle, both being optimum at about $5 \mathrm{~mm}$. This is probably a reflection on the requirements of creatine kinase for the cation.

An alternative approach used by Lowry, Passonneau, Schultz \& Rock (1961) is to cycle twice in two separate operations. In their assay for $\mathrm{NAD}^{+}+\mathrm{NADH}$, for example, they employed a cycle with lactate dehydrogenase as one arm that by virtue of their arrangement produced pyruvate as an accumulating product. They allowed the cycle to turn long enough to give a $10^{3}-10^{4}$-fold yield of pyruvate as compared with the original coenzyme present. Then they added excess of $\mathrm{NADH}$ and lactate dehydrogenase to convert pyruvate into lactate and an equivalent quantity of $\mathrm{NAD}^{+}$was produced. The unwanted NADH was then destroyed with acid and the $\mathrm{NAD}^{+}$assayed as before, giving a final yield of about $10^{7}$-fold. The possibilities are endless in this particular case, and Lowry et al. (1961) claimed to have measured amounts of coenzyme as low as $10^{-15}$ mole at $10^{-6} \mathrm{~mm}$ by this technique, the final pyruvate being measured fluorimetrically by adding NADH and lactate dehydrogenase. Although this type of procedure is somewhat time-consuming, its sensitivity rivals that of radioactive techniques for microanalysis, and there is no reason theoretically why it should not be used for assaying enzymes that feed substrate into a cycle such as is reported in this present work, and hence to make feasible the detection of single enzyme molecules.

The authors thank Computer Projects Ltd. for assistance in programming and donation of computer time.

\section{REFERENCES}

Chappell, J. B. \& Perry, S. V. (1954). Biochem. J. 57, 421. 
Cori, O., Traverso-Cori, A., Lagarrigue, M. \& Marcus, F. Kuby, S. A., Noda, L. \& Lardy, H. A. (1954). J. biol. (1958). Biochem. J. 70, 633.

Chem. 210, 65.

Glock, G. E. \& McLean, P. (1955). Biochem. J. 61, Lowry, O. H., Passonneau, J. V., Schultz, D. W. \& Rock, 381.

M. K. (1961). J. biol. Chem. 236, 2746.

Greenbaum, A. L., Clark, J. B. \& McLean, P. (1965). McQuate, J. T. \& Utter, M. F. (1959). J. biol. Chem. 234, Biochem. J. 95, 161. 2151.

Grisolia, S., Mokrasch, L. C. \& Hospelhorn, V. D. (1958). Biochim. biophys. Acta, 28, 350.

Grunberg-Manago, M. (1963). Progr. Nucleic Acid Res. 1, 98.

Ochoa, S. \& Mii, S. (1961). J. biol. Chem. 236, 3303.

Oliver, I. T. (1954). Biochim. biophys. Acta, 14, 584.

Perry, S. V. (1954). Biochem.J. 57, 427.

Tanzer, H. L. \& Gilvarg, C. (1959). J. biol. Chem. 234, 3201.

Hakala, M. T., Glaid, A. J. \& Schwert, G. W. (1956). J. biol. Chem. 221, 191.

Tietz, A. \& Ochoa, S. (1958). Arch. Biochem. Biophys. 78, 477.

Jandorf, B. J., Klemperer, F. W. \& Hastings, A. B. (1941). J. biol. Chem. 138, 311.

Williams, F. R. \& Grunberg-Manago, M. (1964). Biochim. biophys. Acta, 89, 66. 